\title{
Water Jet Guided Laser vs. Conventional Laser: Experimental Comparison of Surface Integrity for Different Aerospace Alloys
}

\author{
Erdem Bektas $^{* 1,2}$, Levent Subasi ${ }^{1}$, Aydemir Gunaydin ${ }^{1}$, Jeremie Diboine ${ }^{3}$, Ozan Can Ozaner ${ }^{1}$ and Demircan Canadinc ${ }^{2}$ \\ ${ }^{1}$ Tusas Engine Industries (TEI), Eskisehir, Turkey \\ ${ }^{2}$ Advanced Materials Group (AMG), Koc University, Istanbul, Turkey \\ ${ }^{3}$ Synova S.A., Duillier, Switzerland \\ ${ }^{*}$ Corresponding author's e-mail: erdem.bektas@tei.com.tr
}

\begin{abstract}
Laser cutting is a widely used manufacturing technology in aerospace industry. However, although considered a fast process, lasers have some quality issues associated with high heat input to the materials. Water jet guided laser is a technology that overcomes most of these issues, where pressurized water in the process provides focusing, cooling and cleaning on the cut region, eliminating undesired side effects of the laser. In this study, cutting performances of a water jet guided laser system and a conventional laser system were compared. For this purpose, well-known quality problems of laser cutting were considered, namely dross/burr formation, surface quality, taper and recast layer. The samples were chosen among commonly used aerospace alloys: Haynes 188, Inconel 718, Inconel 625, Rene 41 and Ti-6Al-4V. Visual checks and measurements of surface roughness, taper and recast layer were conducted on the samples. The results show that cutting with a water jet guided laser system overall provides better surface integrity when compared to a conventional laser system.
\end{abstract}

DOI: $10.2961 /$ jlmn.2021.01.2001

Keywords: laser cutting, water jet, aerospace materials, surface quality, surface integrity

\section{Introduction}

Aerospace parts are made of high-temperature, highstrength and wear-resistant alloys. Machining such materials using traditional methods is challenging. Therefore, non-traditional production processes are preferred for various applications [1]. Laser cutting is one of the important manufacturing technologies, which is widely utilized in the aerospace industry. There are many parts, such as exhaust liners, baffles, combustion domes and liners, ducts and impingement rings, which are manufactured using lasers. However, lasers might lead to poor quality characteristics, quantified in terms of kerf width, kerf deviation, kerf taper, heat affected zone (HAZ), surface roughness, dross formation, dross height, recast layer, micro cracks and hardness [2].

\subsection{Conventional laser cutting}

There are numerous studies focusing on quality characteristics of conventional laser cutting. Surface integrity is generally reported to be problematic independent of laser type and the processed material, especially when compared to other manufacturing methods. For instance, Holmber et al. [3] compared different non-traditional production processes, namely abrasive water jet machining (AWJM), electrical discharge machining (EDM) and laser beam machining (LBM) in terms of quality characteristics. They performed experiments on a nickel-based aerospace alloy, Inconel 718, and found the kerf surface of LBM cuts to exhibit the poorest surface quality, as shown in Fig. 1.

Yilbas et al. [4] performed laser cutting experiments on stainless steel 304, Inconel 625 super alloy, and titanium alloy (Ti-6Al-4V). The effect of laser power and cutting speed on kerf surface was analyzed. Fig. 2 shows that the kerf surfaces are similar for the alloys considered in this study, such that striations (i.e. ripples) stemming from the flow of liquid metal were observed.
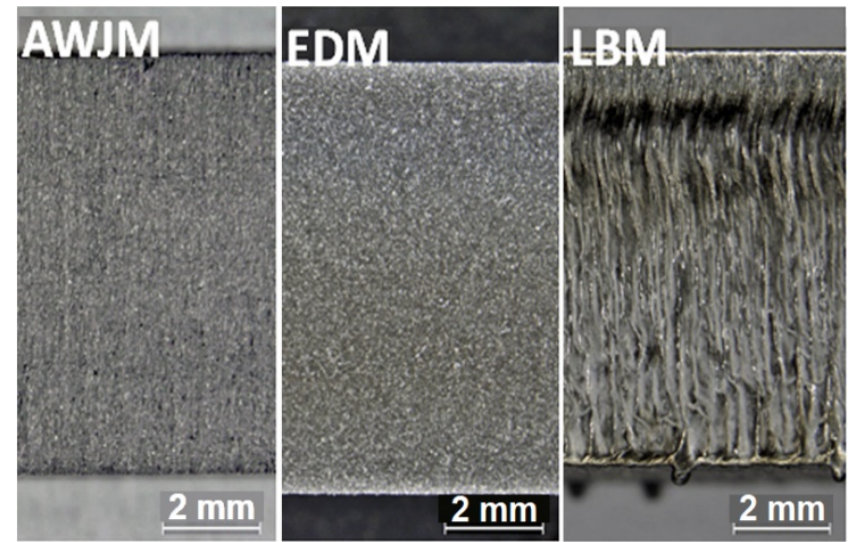

Fig. 1 General appearance of the machined surfaces [3].
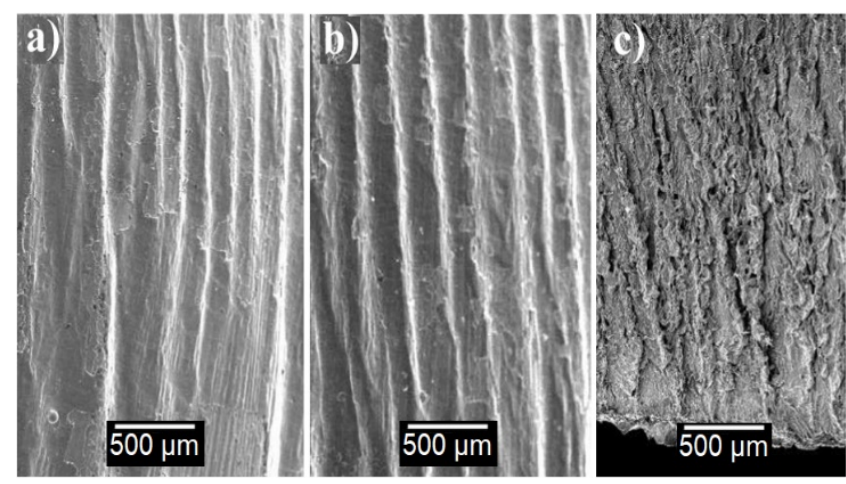

Fig. 2 Laser cut kerf surfaces: (a) stainless steel, (b) Inconel 625, (c) Ti-6Al-4V alloy [4]. 


\subsection{Water jet guided laser cutting}

Taylor [5] showed that a small diameter jet of a transparent liquid having a refractive index greater than the surrounding medium could entrap a light beam and act as an optical fiber. Based on this principle, Richerzhagen [6] invented a method and apparatus for machining materials with a water guided laser beam. The technology was called Water Jet Guided Laser (WJGL) or Laser MicroJet ${ }^{\circledR}$ (LMJ), and made commercially available by the Swiss company Synova SA. Fig. 3a [7] shows the working principle of the process and $3 \mathrm{~b}$ shows the conventional system for comparison purpose. On the WJGL system helium gas is blowed from nozzle tip, flowed around the water jet, and its aim is not to assist laser but to prevent early water droplet formation and to keep proper linear shape of the water jet. The WJGL machining has many advantages over conventional LBM, such as consistent focusing, burr-free cutting, minimized tapering, reduced HAZ and recast layer [8].
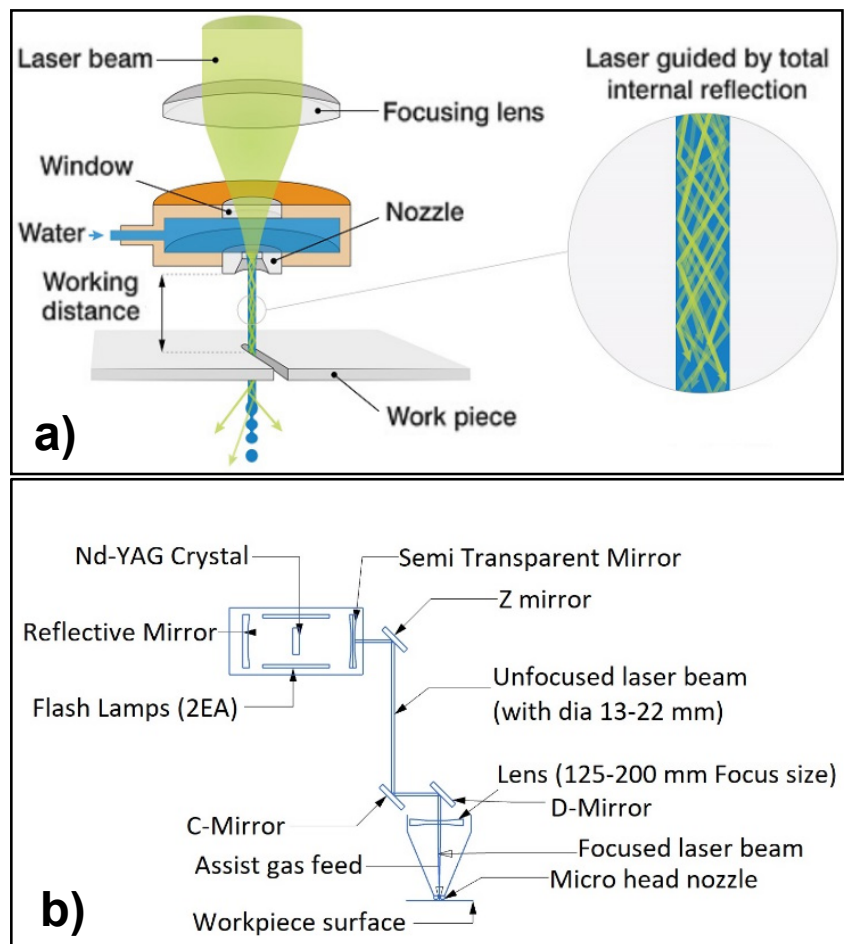

Fig. 3 Working principle of the (a) water jet guided laser, (b) conventional laser beam machining [7].

There are various WJGL cutting studies in the literature for different kind of materials. In one of the previous studies, Mai and Richerzhagen [9] investigated the WJGL cutting capabilities on organic light emitting diode (OLED) masks. They observed precise and high-speed cutting that is free of dross, contamination and thermal stress. It was reported that the WJGL has better quality compared to etching or conventional laser cutting.

Hock et al. [10] compared fiber laser cutting to WJGL cutting on stainless steel and brass sheets. They reported dross, HAZ and varying kerf width for fiber laser cutting. They achieved dross free cuts with no HAZ and small kerf width for the WJGL process. However, process times for the WJGL were considerably higher. It was recommended that
WJGL cutting should be preferred when the work piece is thin and high-quality cuts are required.

Bruckert et al. [11] performed WJGL cutting of different shapes on crystalline solar cells. They reported the cuts to be free of contamination, which led to minimum electrical losses. Eventually, it was concluded that different set of process parameters has to be developed for the specific type of solar cell.

Richmann et al. [12] studied the WJGL cutting of ultrahard diamond tool materials and compared the results to EDM cutting. They reported lower surface roughness and higher process speed with WJGL cutting. The samples had high edge sharpness, which removed the necessity for finish grinding for some applications.

Sun et al. [13] conducted a comparative investigation for conventional laser cutting and WJGL cutting on carbon fiber reinforced plastics (CFRP). The differences of kerf geometry, HAZ, surface morphology and efficiency were discussed. The experiments showed that the HAZ of WJGL is smaller than conventional LBM. However, the process was slower and there was a need for using multiple passes to achieve a complete cut through the sample thickness.

\subsection{Aim of the study}

All of the aforementioned studies have experimentally demonstrated that WJGL cutting is superior in terms of surface integrity. Therefore, this process can be conveniently used for cutting aerospace materials. In this paper, the results of the cutting experiments of a WJGL system and a conventional LBM system are presented and compared. The experiments were performed on aerospace alloys and the results were evaluated in terms of different quality characteristics.

\section{Materials and Methods}

Some of the most commonly used aerospace alloys were selected for the experiments. Basic material information can be seen in Table 1 . The density at room temperature and the thermal diffusivity at $2 / 3$ melt temperature shown in the table were calculated using the commercial software JmatProv9 - the Materials Property Simulation Package.

Table 1 Basic material information

\begin{tabular}{cccc}
\hline Alloy & Base & $\begin{array}{c}\text { Density } \\
\left(\mathrm{g} / \mathrm{cm}^{3}\right)\end{array}$ & $\begin{array}{c}\text { Thermal Diffusivity } \\
\left(\mathrm{mm}^{2} / \mathrm{s}\right)\end{array}$ \\
\hline Haynes 188 & Cobalt & 8.99 & 5.02 \\
Inconel 718 & Nickel & 8.28 & 3.89 \\
Inconel 625 & Nickel & 8.51 & 3.87 \\
Rene 41 & Nickel & 8.28 & 4.07 \\
Ti-6Al-4V & Titanium & 4.41 & 9.00 \\
\hline
\end{tabular}

Thin strips of $0.8 \mathrm{~mm}$ thickness were cut for experimental purposes with a WJGL machine and a conventional laser cutting machine. Each experiment was carried out three times on companion samples to ensure repeatability. WJGL system uses a diode pumped pulsed $532 \mathrm{~nm}$ Nd:YAG laser, whereas the conventional LBM system uses a flash lamp pumped pulsed $1064 \mathrm{~nm} \mathrm{Nd:YAG} \mathrm{laser.} \mathrm{Fig} \mathrm{4.} \mathrm{shows} \mathrm{the} \mathrm{in-}$ stances of the cutting experiments for both systems. 
It was not possible to employ exactly the same set of test parameters for both machines due to their configurational differences. Thus, different sets of process parameters had to be used for each system depending on machine constraints, best practices and preliminary trials. The set of selected parameters can be found in Table 2 .
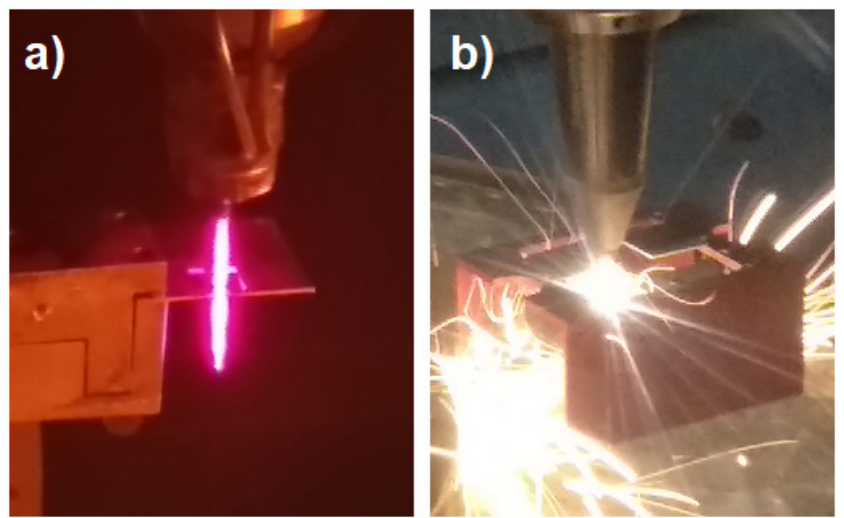

Fig. 4 Cutting experiments: (a) WJGL, (b) LBM

Table 2 Laser and process parameters

\begin{tabular}{lll}
\hline & WJGL & LBM \\
\hline Laser Power & $35 \mathrm{~W}(15 \mathrm{~W})$ & $170 \mathrm{~W}$ \\
Pulse Width & $200 \mathrm{~ns}$ & $0.4 \mathrm{~ms}$ \\
Freq uency & $10 \mathrm{kHz}$ & $120 \mathrm{~Hz}$ \\
Nozzle/Spot Size & $50 \mu \mathrm{m}$ & $0.3 \mathrm{~mm}$ \\
Peak Intensity & $382 \mathrm{MW} / \mathrm{cm}^{2}$ & $5 \mathrm{MW} / \mathrm{cm}^{2}$ \\
Water Pressure & $200 \mathrm{bar}$ & - \\
Assist Gas & Helium $(1 \mathrm{l} / \mathrm{min})$ & Air $(6.2 \mathrm{bar})$ \\
Standoff Distance & $10 \mathrm{~mm}$ & $1.25 \mathrm{~mm}$ \\
Feed & $250 \mathrm{~mm} / \mathrm{min}$ & $250 \mathrm{~mm} / \mathrm{min}$ \\
\hline
\end{tabular}

Even though the laser power of WJGL was set to $35 \mathrm{~W}$ for the experiments, there were some transmission losses due to optics, water, etc. Measurements showed that the power on the work piece surface is $15 \mathrm{~W}$ at $10 \mathrm{~mm}$ stand-off distance, and the peak intensity was calculated accordingly.

The experiments showed that the conventional LBM system was able to make the cuts with a single pass. However, it was only possible to cut the coupons with multiple passes using the WJGL machine. Depending on the material, the cutting toolpath was employed five to seven times until the cut was completed. Even though the feed was the same for both machines, WJGL processing time was longer because of this multi-pass method. Some of the difference in cutting time is considered to be linked to the laser power levels at which each laser unit operates. There is also the difference between the ablation mechanisms. Looking at the peak intensity levels, LBM mostly melts the material and ejects the melt through the kerf, whereas WJGL removal mechanism is vaporization. This creates a difference in efficiency between the two systems. The ablation mechanism of WJGL is discussed in more detail in Section 4.

\section{Results and Discussion \\ 3.1 Surface quality}

Examples of the microscopy images of the cut surfaces are shown in Fig. 5. The striation effect created by the conventional laser perpendicular to the cutting direction can be clearly seen in the photos. Furthermore, there was also dross formation at the exit side for all the LBM cut samples. The maximum dross height was measured for each coupon and the corresponding average values are presented in Table 3. Inconel 625 has the least dross, whereas other materials gave similar results. The WJGL cuts showed only noncontinuous, distanced, singular, around $\sim 1-3 \mu \mathrm{m}$ height drosses as Fig.6, but there are horizontal marks due to the multi-pass strategy.
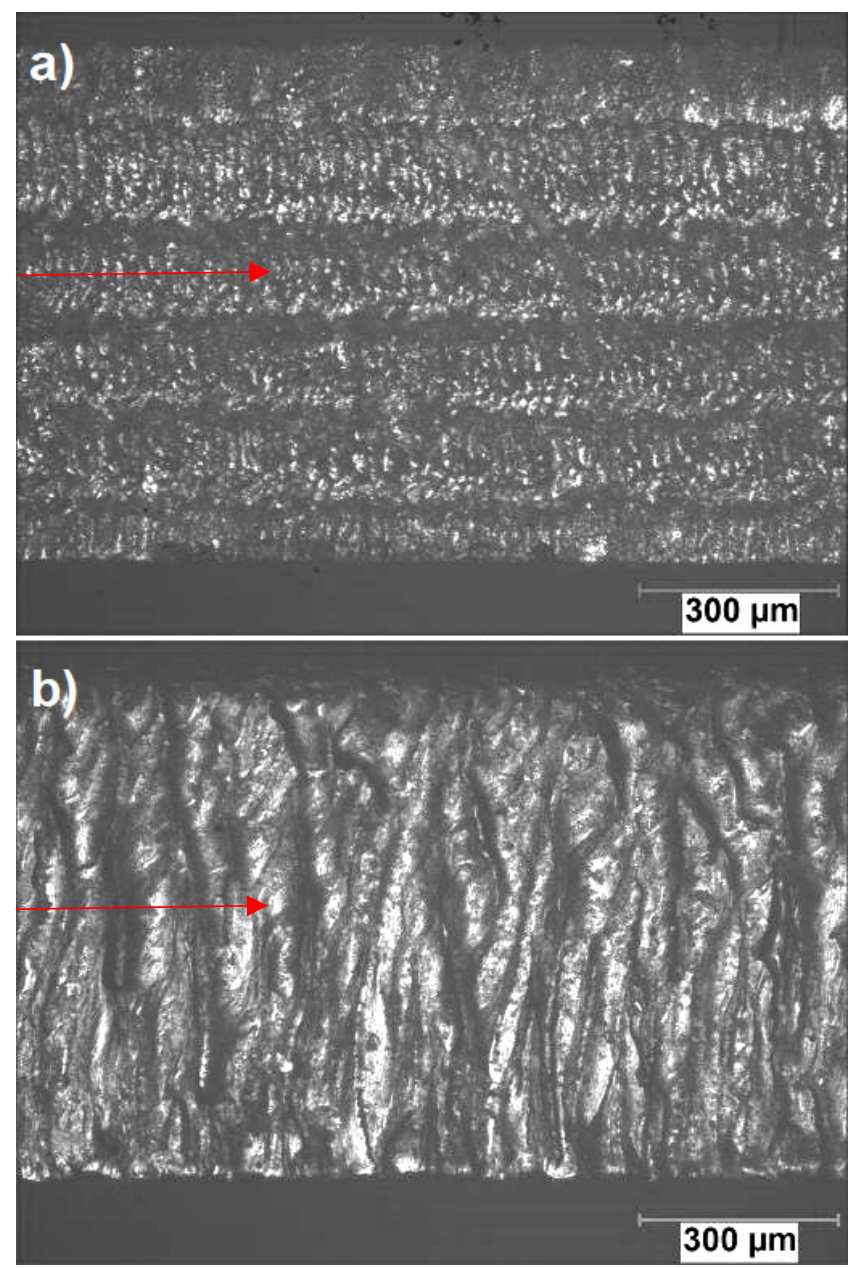

Fig. 5 Optical microscopy images of the Inconel 625samples cut with: (a) WJGL, (b) LBM (red arrow: surface roughness measurement direction)

Table 3 Dross average heights for LBM cut samples (mm)

\begin{tabular}{lc}
\hline & Dross height \\
\hline Haynes 188 & 0.055 \\
Inconel 718 & 0.049 \\
Inconel 625 & 0.023 \\
Rene 41 & 0.061 \\
Ti-6Al-4V & 0.050 \\
\hline
\end{tabular}




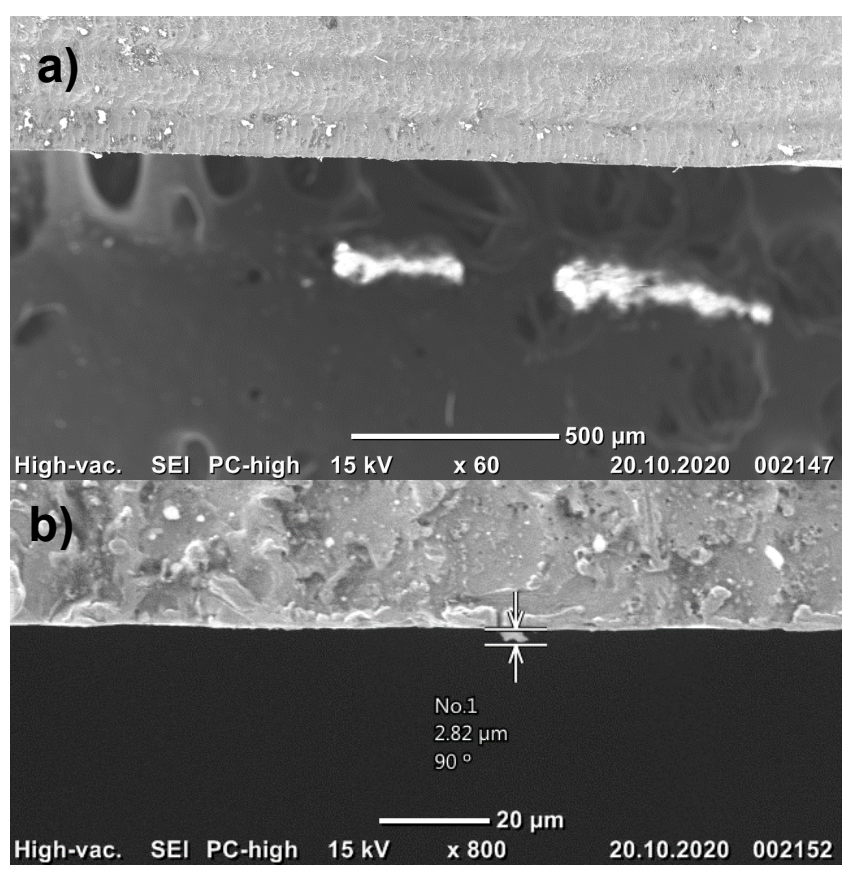

Fig. 6 SEM images of the Rene 41 samples cut with WJGL: (a) 60x, (b) $800 x$

$\mathrm{Ra}$ and $\mathrm{Rz}$ surface roughness measurements were made with Marsurf SD26 measurement system. Surface roughness measurement direction is the same with laser cut movement direction. Four measurements were taken from different regions on each sample. The average results for each material and laser system are shown separately in Table 4. Based on the measurements, the surface quality comparison plot shown in Fig. 7 can be drawn.

Table 4 Average surface roughness $(\mu \mathrm{m})$

\begin{tabular}{lcccc}
\hline & \multicolumn{2}{c}{ WJGL } & \multicolumn{2}{c}{ LBM } \\
\hline & $\mathrm{Ra}$ & $\mathrm{Rz}$ & $\mathrm{Ra}$ & $\mathrm{Rz}$ \\
\hline Haynes 188 & 1.11 & 7.41 & 2.91 & 17.01 \\
Inconel 718 & 1.24 & 8.19 & 3.29 & 18.33 \\
Inconel 625 & 1.30 & 8.72 & 3.26 & 20.22 \\
Rene 41 & 1.29 & 8.26 & 2.78 & 16.37 \\
Ti-6A1-4V & 2.31 & 13.84 & 3.53 & 20.44 \\
\hline
\end{tabular}

It can be seen from the results that WJGL has significantly better surface quality as compared to LBM. This is expected, since there is no ripple formation on the WJGL cut surfaces. It is also observed that Ti-6Al-4V alloy has the worst surface quality when compared to other materials, which is further discussed in Section 4.

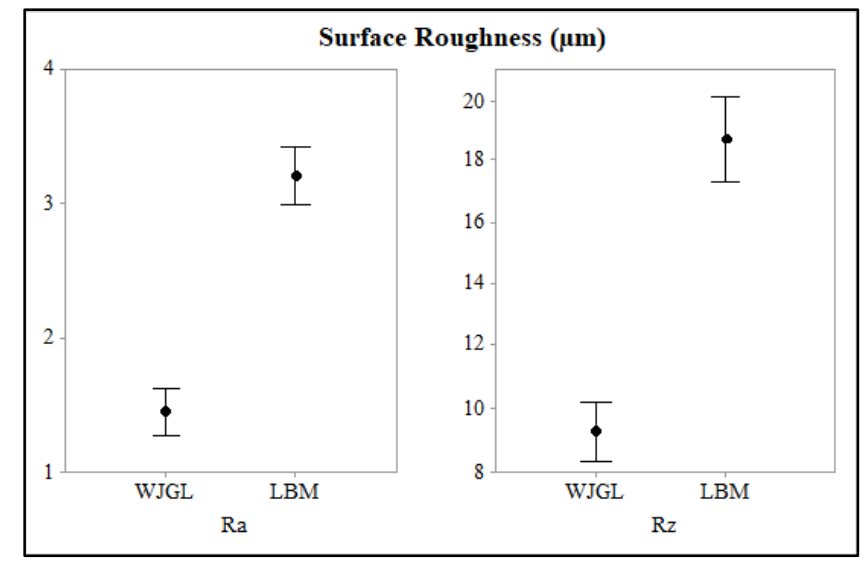

Fig. 7 Surface quality comparison plots for WJGL and LBM

\subsection{Taper}

Kerf taper measurements were carried out with an optical microscope, taking the cross-section of the samples perpendicular to the cutting direction. The taper angle was calculated geometrically by taking the inverse tangent of the length difference between the top and the bottom sides divided by the sample thickness. The average results for each material and laser system are listed separately in Table 5. The worst taper achieved for the WJGL and LBM is shown in Fig. 8. It can be seen from the results that WJGL has significantly less taper as compared to LBM, which is attributed to the focusing ability of the water jet. Additionally, taper doesn't seem to depend on material properties.

Table 5 Average kerf taper $\left({ }^{\circ}\right)$

\begin{tabular}{lcc}
\hline & WJGL & LBM \\
\hline Haynes 188 & 1.8 & 8.5 \\
Inconel 718 & 1.3 & 7.6 \\
Inconel 625 & 1.4 & 8.7 \\
Rene 41 & 1.7 & 8.8 \\
Ti-6Al-4V & 1.2 & 8.3 \\
\hline
\end{tabular}
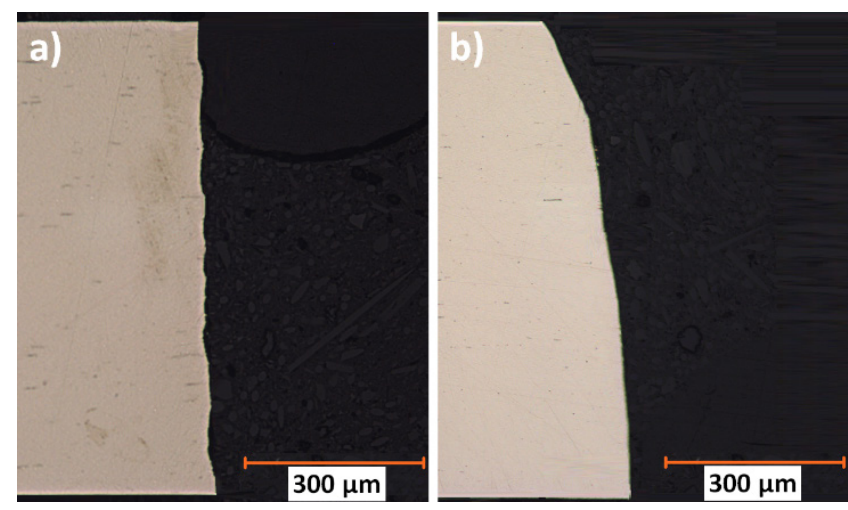

Fig. 8 Kerf taper of Hayness 188: (a) WJGL, (b) LBM

\subsection{Recast layer}

The samples were etched with an acid solution to reveal the recast layer. Then, measurements were taken using a metallographic microscope. The average results for each material and laser system are tabulated separately in Table 6. 
Examples of the microscopy images of the cut surfaces are shown in Fig. 9.

$50 \mu \mathrm{m}$ below recast layer is generally acceptable for most of the aerospace parts. With its recast layer below 9 $\mu \mathrm{m}$, water jet guided laser machining is suitable to be used in more critical parts without any post machining need when compared to LBM.

Table $6 \quad$ Average recast layer $(\mu \mathrm{m})$

\begin{tabular}{lcc}
\hline & WJGL & LBM \\
\hline Haynes 188 & 2.4 & 15.7 \\
Inconel 718 & 1.7 & 16.2 \\
Inconel 625 & 2.0 & 13.9 \\
Rene 41 & 2.0 & 13.2 \\
Ti-6Al-4V & 8.7 & 19.8 \\
\hline
\end{tabular}
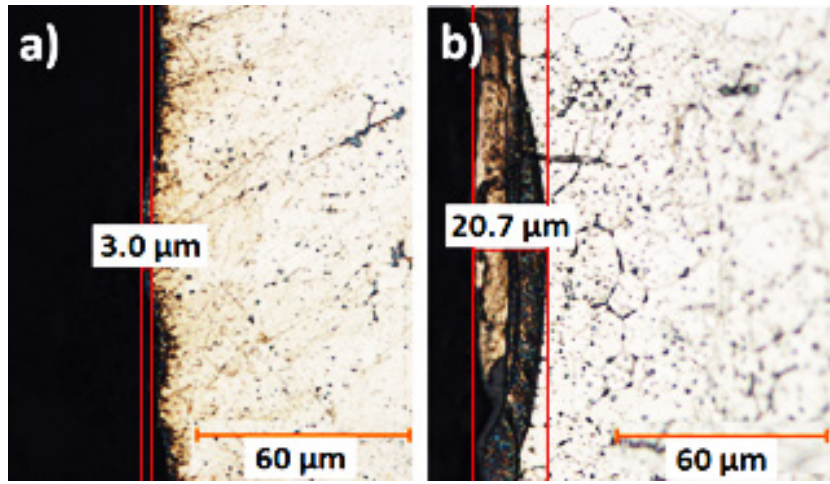

Fig. 9 Recast layer of Hayness 188: (a) WJGL, (b) LBM

It can be seen from the results that WJGL has significantly less recast layer as compared to LBM. There are several reasons for this observation. First, the water jet cools down the work piece within each pulse. There is some heat accumulation, but there is enough time between consecutive laser pulses for the water jet to take away most of the residual heat of ablation. Then, LBM has a Gaussian beam profile, whereas WJGL has a flat top beam profile in the water jet. There is also spot size difference between the two systems. Thus, the outer edge of the beam spot is larger and has less energy for LBM cutting, which causes to melt the material more. Another reason is the water jet cleaning effect. Specifically, high-pressure water removes most of the molten material instantly from the cut region, limiting drastically the re-solidification on the sidewalls. Another observation is that the Ti-6Al-4V alloy has the most recast layer when compared to other materials, which is further discussed in Section 4.

\section{Material Removal Mechanism for WJGL}

The main disadvantage of WJGL is the high processing time for the used parameter set when compared to LBM. Although WJGL has higher peak intensity, its cutting depth is less. The WJGL process mostly vaporizes the material, which takes up more energy to accomplish. Some simplifying assumptions are made to calculate the cut depths:

- One pulse of laser cuts a cylindrical shape with a base area of $A$ from the surface.
- All the laser energy is absorbed in the cylindrical volume and no heat transfer to the surrounding material occurs, i.e. thermal penetration depth is neglected.

- Material is removed only by vaporization, i.e. no melting or ionization occurs.

Cut depth of a single pulse can be calculated using an energy balance approach [14] as in Formula (1):

$$
\begin{array}{ll}
\text { One Pulse Ablation Depth }=\frac{E_{p} \times \text { Absorption Coef. }}{A . \rho . \Delta H} \\
\begin{array}{ll}
E_{p} & : \text { Pulse energy }(\mathrm{J}) \\
A & : \text { Cut area for one pulse }\left(\mathrm{cm}^{2}\right) \\
\rho & : \text { Material density }\left(\mathrm{g} / \mathrm{cm}^{3}\right) \\
\Delta H & \text { : Room Temperature to Vaporization Tem- } \\
& \text { perature Enthalpy }(\mathrm{J} / \mathrm{g})
\end{array}
\end{array}
$$

In this formulation, considering the reflectivity of the materials, absorption coefficients were taken as 0.35 for the cobalt alloy Haynes 188 [15], 0.4 for all the nickel alloys [16], and 0.2 for Ti-6Al-4V [17]. Cut area was calculated based on the results of the preliminary studies on the WJGL system. The cut area diameter was taken as $60 \mu \mathrm{m}$. Each alloy's enthalpy is a summation of:

- The enthalpy of the alloy up to the liquidus melting temperature, calculated with the JmatPro software,

- The elemental enthalpies from liquidus temperature, calculated with the JmatPro software, to the elements' vaporization temperature [18], and

- Vaporization enthalpies of the major compound elements [18] that are in the chemical composition of the alloy.

The enthalpies are approximately calculated as given in Table 7.

Table 7 Enthalpy difference from room temperature to vaporization point $\left(\mathrm{J} / \mathrm{g}\right.$ and $\left.\mathrm{J} / \mathrm{mm}^{3}\right)$

\begin{tabular}{lcc}
\hline & $\Delta \mathrm{H}(\mathrm{J} / \mathrm{g})$ & $\Delta \mathrm{H}\left(\mathrm{J} / \mathrm{mm}^{3}\right)$ \\
\hline Haynes 188 & 8594 & 77.26 \\
Inconel 718 & 9024 & 74.72 \\
Inconel 625 & 8938 & 76.06 \\
Rene 41 & 9202 & 76.19 \\
Ti-6Al-4V & 12105 & 53.38 \\
\hline
\end{tabular}

The pulse energy is calculated using Formula (2) [19]:

$$
E_{p}=\frac{\text { Laser Power }}{\text { Pulse Frequency }}
$$

Using Formulae (1), (2), and conversion from $\mathrm{cm}$ to $\mathrm{mm}$, one-pulse cutting depths are calculated for each alloy, as listed in Table 8. 
Table 8 Theoretical one-pulse cut depth (mm)

\begin{tabular}{lc}
\hline & Depth $(\mathrm{mm})$ \\
\hline Haynes 188 & 0.00240 \\
Inconel 718 & 0.00284 \\
Inconel 625 & 0.00279 \\
Rene 41 & 0.00279 \\
Ti-6Al-4V & 0.00199 \\
\hline
\end{tabular}

These values are the cut depths that can be achieved by a single pulse depending on the material properties, and for cylindrical material removal with only vaporization approach. However, depending on the frequency and feed rate values, the overlap ratio is greater than $99 \%$ for two consequent pulses during cutting. A single spot on the cutting axis on surface receives about 120 pulses, which means that a 0.8 $\mathrm{mm}$ plate should be cut thoroughly only with three or four passes, whereas experiments were completed with five to seven passes. This fact proves there is an efficiency loss in the process as of yet unidentified. The laser energy density might be reduced at the cut region or the laser beam might be blocked decreasing the material removal rate. This finding doesn't seem to be related to water jet splash back, since the established kerf ejects the water away from the cut region as the jet progresses and the light guided through the jet is not disturbed, as illustrated in Fig. 10.

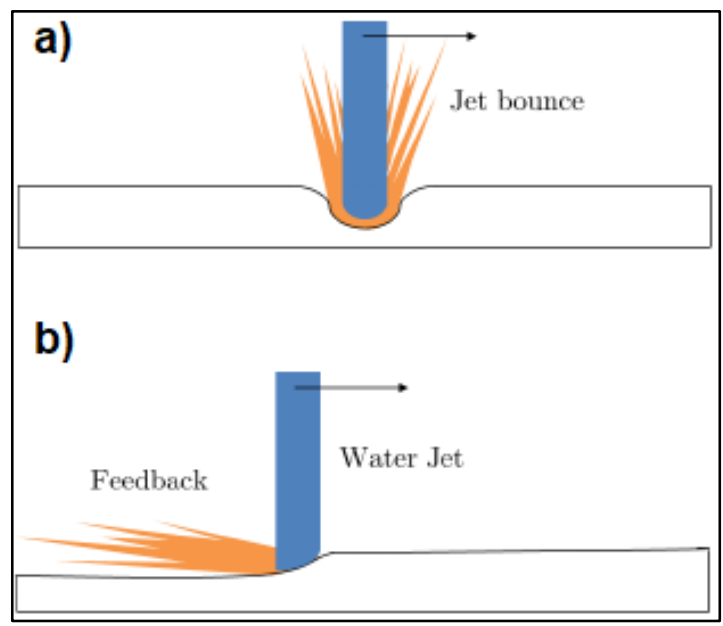

Fig. 10 (a) Starting Kerf - Water ejection directed against the jet, (b) Established Kerf - Water ejection directed away

In order to calculate the efficiency of the process, the weight of the removed material should be measured and compared to the theoretical calculations. Efficiency can be described as the ratio of experimental weight loss to theoretically calculated weight loss, as shown in Formulae (3) and (4):

$$
\text { Process Efficiency }=\frac{\text { Measured Weight Loss }}{\text { Calculated Weight Loss }}
$$

Calculated Weight Loss $=\frac{P . l \times \text { Absorption Coef } \text {. }}{F . \Delta H}$
$F$

$$
\text { : Feed rate }(\mathrm{mm} / \mathrm{min})
$$

Further experiments were performed on Rene 41 alloy to calculate the process efficiency for the same parameters given in Table 2. The laser was passed only one time on the surface for making a trench. The weight loss was measured as $0.0017 \mathrm{~g}$ for the obtained cut length. With the necessary unit conversions, equations (3) and (4) give the process efficiency as $52.6 \%$. Thus, some of the laser energy on the cut region seems to be lost. The reason for the overall loss measured for all the materials might be the plasma absorption by inverse Bremsstrahlung and/or energy density reduction on the cut area. Even though most of the incoming laser light is guided to the material through the water jet, the remaining of it might be redirected inside the jet plume causing some of the energy to be lost. It should also be noted that the real process efficiency should be slightly lower than calculated for all materials due to partial melting of material as evidenced by the recast layer measurements, which was neglected for calculation purposes. Furthermore, the efficiency value is not a constant as it is dictated by the process and laser parameters, and material properties.

The results observed on Ti-6Al-4V alloy seem to require further analysis, as it seems to be an outlier in terms of the material removal calculated with the current method. This alloy's necessary energy to heat from room temperature to vaporization point is the lowest per volume. However, it has the lowest absorptivity at $532 \mathrm{~nm}$ wavelength, when compared to other alloys. Thus, it has the least calculated cut depth. The current experiments seem to contradict this assumption, as seen in Table 9, where the average one-pass depth for Ti-6Al-4V is higher than that for the other alloys.

Table $9 \quad$ Experimental cut depths (mm)

\begin{tabular}{lc}
\hline & Average one-pass depth $(\mathrm{mm})$ \\
\hline Haynes 188 & 0.115 \\
Inconel 718 & 0.134 \\
Inconel 625 & 0.131 \\
Rene 41 & 0.133 \\
Ti-6Al-4V & 0.181 \\
\hline
\end{tabular}

Assuming that one spot on the surface receives 120 pulses, as previously mentioned, the real depth per pulse can also be calculated using the average experimental one-pass depth values. Thus, the experimental one-pulse depth values are obtained as shown in Table 10. Comparing the actual one-pulse depths to theoretically calculated depths from Table 8, the efficiency of the process based on this approach can be calculated. The efficiency increase for Ti-6Al$4 \mathrm{~V}$ alloy can also be seen here.
$P$

: Laser power on surface (W)

$l$ 
Table 10 Experimental pulse depths ( $\mathrm{mm})$ and their difference to calculated pulse depths

Experimental one- Real vs. theoretical pulse depth $(\mathrm{mm})$ pulse depth (ratio)

\begin{tabular}{lll}
\hline Haynes 188 & 0.0010 & $41.7 \%$ \\
Inconel 718 & 0.0011 & $38.7 \%$ \\
Inconel 625 & 0.0011 & $39.4 \%$ \\
Rene 41 & 0.0011 & $39.4 \%$ \\
Ti-6Al-4V & 0.0015 & $75.4 \%$ \\
\hline
\end{tabular}

The reason for this finding might be related to the exothermic reaction of Ti-6Al-4V. Specifically, oxide formations of titanium, vanadium and aluminum provide more energy than oxidations of nickel, chrome, cobalt and iron [20], such that this additional energy emission might improve the cutting performance. The oxide layer might also have affected the absorptivity of the alloy. Furthermore, thermal diffusivity of Ti-6Al-4V is greater than that of other alloys, which would result in a higher thermal penetration depth, which was neglected in the simplified material removal model. Thus, higher thermal diffusivity, additional oxidation energy and increase in absorptivity may favor material removal for Ti-6Al-4V and cause rougher surface quality and higher recast layer, as well.

\section{Conclusion}

An experimental study was performed on WJGL and LBM cutting of different aerospace materials. The tests revealed that the overall surface integrity is much better with the WJGL system. No vertical striation and only discrete micrometer level few drosses was observed. Additionally, surface roughness, kerf taper and recast layer characteristics are significantly lower than LBM cutting.

Nevertheless, with the present machine setup, the process has been shown to be slower with WJGL compared to LBM. When the laser energy and alloy enthalpies are considered, it is estimated that WJGL should be able to cut through a $0.8 \mathrm{~mm}$ thick material with a smaller number of passes than was experimentally required. It is concluded that this is likely due to plasma shielding and some of the laser energy being lost in the water plume, causing less energy reaching to the ablation front. The efficiency was calculated from weight loss and geometric approach separately, thus confirming the results. The weight loss approach showed slightly higher efficiency overall. The difference between the efficiency estimated with the two approaches can be explained with the assumptions regarding enthalpy values, cylindrical volume removal and ignoring the thermal penetration depth.

When the results are compared for different materials, it is seen that Ti-6Al-4V alloy has the worst surface roughness and the highest recast layer. The cutting efficiency is also better. One reason for that might be the thermo-physical properties of the material. In particular, its thermal diffusivity is higher than the other alloys, whereas the necessary ablation energy per volume is lower. Furthermore, titanium can oxidize easier than cobalt or nickel alloys and this instant burning can provide additional energy to the process. The oxide layer might also lead to increased absorptivity of the surface.
The following activities are planned as future work in order to investigate better the material removal rate and its correlation to surface integrity:

- High-speed camera monitoring to investigate water jet dynamics and flow modeling,

- Thermo-chemical reaction calculation of oxides for understanding energy transfers and estimating plasma shielding,

- Material characterization on cut surfaces (chemical composition, hardness, corrosion resistance etc.)

- Investigation of the effects of different WJGL process and laser parameters on aerospace alloys, and

- Optimization of the WJGL cutting.

\section{Acknowledgments and Appendixes}

The authors would like to thank to Ronan Martin (Synova S.A.) for his technical support and colleagues from TEI who made this study possible.

\section{References}

[1] F. Klocke, A. Klink, D. Veselovac, D. K. Aspinwall, S. L. Soo, M. Schmidt, J. Schilp, G. Levy, and J.P. Kruth: CIRP Annals, 63, (2014) 703.

[2] A. Sharma and V. Yadava: Opt. Laser Technol., 98, (2018) 264.

[3] J. Holmberg, J. Berglund, A. Wretland, and T. Beno: Int. J. Adv. Manuf. Tech., 100, (2019) 1575.

[4] B. S. Yilbas, M. M. Shaukat, and F. Ashraf: Opt. Laser Technol., 93, (2017) 67.

[5] G. W. Taylor: Appl. Opt., 11, (1972) 786.

[6] B. Richerzhagen: U.S. Patent 5,902,499 (1999).

[7] www.synova.ch/technology/laser-microjet.html (last accessed 08.04.2020).

[8] B. Richerzhagen: The Laser User Magazine, 28, (2002) 28.

[9] T. A. Mai and B. Richerzhagen: SID Int. Symp. Dig. Tec., 38, (2007) 1596.

[10] K. Hock, B. Adelmann, and R. Hellmann: Phys. Procedia, 39, (2012) 225.

[11]F. Bruckert, E. Pilat, P. Piron, P. Torres, B. Carron, B. Richerzhagen, M. Pirot, and R. Monna: Proc. SPIE, Vol 8239, (2012) 82390P.

[12]A. Richmann, S. Kurzen, B. Carron, and B. Richerzhagen: Proc. LIM 2015, (2015) \#15-180.

[13]D. Sun, F. Han, W. Ying, and C. Jin: Proc. CIRP, 71, (2018) 71.

[14]J. F. Ready: "LIA Handbook of Laser Materials Processing", (Magnolia Publishing, Inc., 2001) p.473.

[15] T. Makino, H. Kawasaki, and T. Kunitoo: Bull. JSME, 25, (1982) 804.

[16] J. F. Ready: "Industrial Applications of Lasers", (Academic Press, 1997) p.320.

[17]T. J. Wieting and J. T. Schriempf: J. Appl. Phys., Vol. 47 (1976) 4009.

[18] Y. Zhang, J.R.G. Evans, and S. Yand: J. Chem. Eng. Data, 56, (2011) 328.

[19]C. Y. Yeo, S. C. Tam, S. Jana, and M. W. Lau: J. Mater. Process. Technol., 42, (1994) 15.

[20] S. I. Sandler: "Chemical, Biochemical, and Engineering Thermodynamics", (Wiley, 2017) p.778.

(Received: June 25, 2020, Accepted: December 20, 2020) 